\title{
Transcatheter Aortic Valve Replacement After Coronary Artery Bypass Graft Is Associated With Increased Pacemaker Implantation but Not Reduced Overall Survival
}

\author{
Brent Klinkhammer
}

\begin{abstract}
Background: A history of coronary artery bypass graft (CABG) is a common compelling indication for transcatheter aortic valve replacement (TAVR). However, there is little data on how these patients compare to other TAVR patients. In this study, the short and long-term outcomes of these TAVR patients after CABG are defined.

Methods: A retrospective chart review case-control study of 337 consecutive patients who underwent a TAVR for severe aortic stenosis at Sanford Health in Fargo ND was performed to determine if a history of prior CABG was associated with worse outcomes after TAVR as compared to a TAVR cohort without a history of CABG.
\end{abstract}

Results: Despite higher predicted surgical risk, patients with a history of CABG had no significant difference overall survival at 1 month $(98 \%$ vs. $93 \%, \mathrm{P}=0.112), 6$ months $(94 \%$ vs. $87 \%, \mathrm{P}=0.094), 1$ year $(85 \%$ vs. $77 \%, \mathrm{P}=0.206)$ or 2 years $(70 \%$ vs. $57 \%, \mathrm{P}=0.135)$ post-TAVR. However, a history of CABG was associated with an increase in post-TAVR permanent pacemaker (PPM) implantation (15\% vs. $6 \%, \mathrm{P}=0.015)$.

Conclusions: This study gives evidence to suggest that patients with a history of prior CABG do not have any difference in overall survival as other TAVR patients, despite higher predicted surgical risk and differences in preprocedural comorbidities. Our study also confirms the safety of TAVR in this specific population in lower volume centers.

Keywords: Coronary artery bypass graft; Transcatheter aortic valve replacement; Outcomes; Pacemaker; Survival

\section{Introduction}

The minimally invasive nature of transcatheter aortic valve replacement (TAVR) has made this procedure an attractive option for patient with symptomatic aortic stenosis with elevated

Manuscript submitted January 23, 2018, accepted January 29, 2018

University of Nebraska Medical Center, 982055 Nebraska Medical Center, Omaha, NE 68198-2055, USA. Email: brent.klinkhammer@unmc.edu surgical risk. Many factors increase the risk associated with surgical aortic valve replacement (SAVR) including porcelain aorta, prior sternotomy, prior chest irradiation, severe chest deformity, medical frailty, significant lung disease, and cirrhosis. But perhaps the strongest contraindication to SAVR is a history of coronary artery bypass graft (CABG) surgery. Previous studies have shown that cardiac reoperation is associated with an increase in perioperative myocardial infarctions, lowoutput heart failure, and death [1]. CABG is particularly high risk secondary to iatrogenic changes in anatomy such as a left internal mammary artery that crosses the midline.

Multiple studies have shown TAVR to be a superior option in high or intermediate risk surgical candidates [2, 3]. However there is conflicting evidence in the post-CABG population in regard to TAVR outcomes. For instance, Greason et al found that in comparison to SAVR, TAVR was associated with a nonstatistical trend toward greater all-cause mortality and a significant increase rehospitalization. In this study, TAVR was associated with a $36.1 \%$ death rate at 2 years [4]. On the other hand, studies by Nguyen et al and others have shown similar to improved outcomes with TAVR in this specific population $[5,6]$.

There is however a paucity of data on how patients with a history of prior CABG compare to other TAVR patients in terms of periprocedural and long-term outcomes. Historically, patients with history of CABG have increased mortality following major vascular procedures [7]. There may be clinically significant differences in procedural complications and clinical consequences that may be avoidable and potentially intervenable in the periprocedural and postprocedural period.

Furthermore, the published research on the outcomes and safety TAVR in patients with a history of CABG has all been from larger urban medical centers. These studies were conducted mainly at large volume centers which traditionally have more experience and better surgical outcomes for these advanced procedures. It is vital that the safety of TAVR in his particular patient population be also established in smaller, more rural surgical center as well. Our objective in this study was to both further establish the safety and acceptability of TAVR in patients with a history of CABG and demonstrate that post-TAVR outcomes from a smaller surgical center are comparable to the outcomes from urban medical centers.

\section{Methods}

A single institution retrospective cohort study was conducted. 
Table 1. Baseline Characteristics

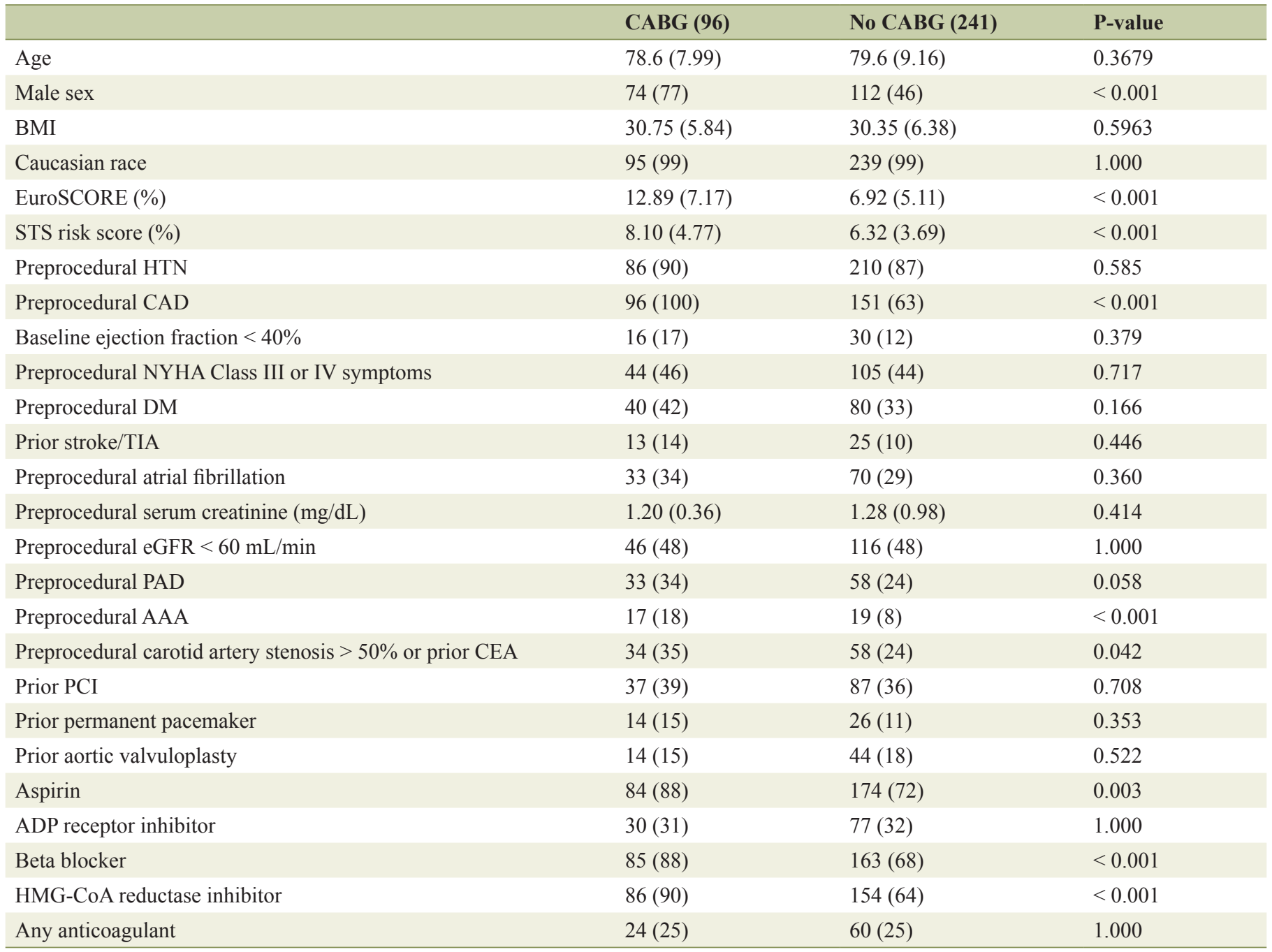

Values are mean (standard deviation) or $\mathrm{n}(\%)$.

We performed a retrospective chart review of 337 consecutive patients who underwent TAVR at Sanford Health in Fargo, ND from August 10, 2012 to November 15, 2016 for severe aortic stenosis, defined as an aortic valve area less than $1 \mathrm{~cm}^{2}$. The last date of data acquisition was January 4, 2017. The entire cohort was divided in two groups where the patients with a history of CABG were placed in one cohort and all other patients were designated as controls. Primary outcomes were overall survival at 1 month, 6 months, 1 year, and 2 years post-TAVR. Secondary outcomes were procedural complications, post-TAVR permanent pacemaker (PPM) implantation, major adverse cardiovascular and cerebrovascular events (MACCE) defined as death from any cause, myocardial infarction, rehospitalization, or stroke, cardiovascular mortality, myocardial infarction, stroke/transient ischemic attack (TIA), heart failure exacerbation, or rehospitalization for any reason in defined time periods. Preprocedural, $24 \mathrm{~h}$ postprocedural, and 1 year postprocedural echocardiographic data were also compared. The clinical outcomes were assessed in accordance with the standardized end- point definitions for TAVR of the Valve Academic Research Consortium-2 [8]. Heart failure exacerbation was defined as a gradual or rapid change in heart failure signs and symptoms resulting in a need for a change in therapy or hospitalization.

Informed consent was not required for inclusion in our retrospective study due to the nature of the study, and the absence of any direct interventions. This study protocol received dual IRB approval from the University of North Dakota IRB and from the Sanford Health IRB. The Fisher's exact test was performed to determine statistical significance of categorical data and $t$-test or Wilcoxon two-sample test were used to determine the statistical significance continuous variables. All P-values were two-sided, and P-values $<0.05$ were considered significant.

\section{Results}

A total of 96 of the 337 patients reviewed had a history of 
Table 2. Procedural Characteristics

\begin{tabular}{llll}
\hline & CABG $(\mathbf{n = 9 6 )}$ & No CABG $(\mathbf{n}=\mathbf{2 4 1})$ & P-value \\
\hline Approach & & & 1.000 \\
Transfermoral & $78(81)$ & $195(81)$ & 0.611 \\
Transapical & $16(17)$ & $34(14)$ & 0.678 \\
Transaortic & $1(1)$ & $6(2)$ & 0.189 \\
Trans-subclavian & $0(0)$ & $6(2)$ & 0.285 \\
Transcaval & $1(1)$ & $0(0)$ & 0.276 \\
Mean LOS after TAVR (days) & $4.1(5.04)$ & $5.1(7.91)$ & 0.788 \\
Valve type & & & 1.000 \\
First generation Sapien & $25(26)$ & $67(28)$ & 0.523 \\
Sapien XT & $14(15)$ & $37(15)$ & 0.882 \\
Sapien S3 & $35(36)$ & $78(32)$ & 1.000 \\
First generation CoreValve & $19(20)$ & $51(21)$ & 0.0498 \\
CoreValve Evolute & $3(3)$ & $8(3)$ & $25.7(2.69)$ \\
Mean valve size (mm) & $26.4(2.58)$ & \\
\hline
\end{tabular}

Values are mean (standard deviation) or $\mathrm{n}(\%)$.

CABG prior to TAVR. Baseline characteristics for both groups are given in Table 1. Several statistically significant differences were noted in sex, STS risk score, EuroSCORE, preprocedural coronary artery disease (CAD), preprocedural abdominal aortic aneurysm (AAA). There were also slight differences in the utilization of several cardiovascular pharmacological agents including aspirin, beta blockers, and statins. There was a high amount of significant comorbidities in both groups including a $73 \%$ prevalence of CAD in the entire cohort. Mean age of the entire cohort was 79.3 years of age. Procedural characteristics for both groups are given in Table 2. There was no statistical differences in the specific type of valve used, however there was small, but statistical significantly difference in mean valve size. Pre- and postprocedural echocardiographic data are given in Table 3. Differences in valve area, peak aortic velocity, peak and mean aortic gradient, and ejection fraction were noted at baseline. Patients with a history of CABG had a lower mean ejection fraction, but slight less aortic valve obstruction than controls. The baseline difference in ejection was sustained directly following the procedure, and patients with prior CABG had less of ejection fraction improvement at $24 \mathrm{~h}$ post-TAVR. The difference in ejection fraction at 1 year following the procedure was not significant.

Finally, the primary and secondary outcomes data for this study are given in Table 4 . There was a nonsignificant trend towards improved overall survival in the CABG cohort. However, a history of prior CABG was associated with a statistically significant increase in need for post-TAVR PPM implantation. No other significant differences in study outcomes were noted between the two groups.

\section{Discussion}

This study further characterizes the efficacy and safety of
TAVR in patients with a history of severe aortic stenosis and prior CABG and gives evidence to suggest that outcomes from lower volume centers are equitable to the previous published multicenter studies. Furthermore, although this study does not directly compare TAVR outcomes to SAVR outcomes, this study helps dispel concerns about an excessive risk of increased overall mortality with TAVR after CABG and demonstrates clinically acceptable outcomes in this specific population.

Importantly, our study also establishes the clinical safety of a TAVR in patient with prior CABG. As previously noted, concerns about the safety of major vascular interventions after $\mathrm{CABG}$ were formerly warranted. However, we found no difference in overall survival between the two cohorts in this study and actually found a nonsignificant trend towards improved overall survival. This trend is remarkable given the larger risk surgical risk and increased burden of significant comorbidities at baseline. For instance, our prior $\mathrm{CABG}$ cohort did have a much larger proportion of male patient which has been associated with increased mortality after TAVR in some studies [9]. As evidence in lower surgical risk population evolves, comparisons between prospectively defined TAVR patient groups will become of increased clinical importance and define clinical indication for post-TAVR follow-up.

This study did find a significant increase in post-TAVR PPM implantation after TAVR in post-CABG patients. To our knowledge, this is the first study to document an increased risk in TAVR-associated PPM in a specific and non-electrophysiologically defined patient population. This risk could potentially be reduced by high valve positioning, which has been associated with lower risk of PPM [10]. Frequent electrocardiogram (EKG) monitoring in immediate and short-term follow-up period in this specific patient population may be indicated.

The noninferiority of survival outcomes in the prior CABG cohort in our study despite higher EuroSCORE and 
Table 3. Echocardiographic Data

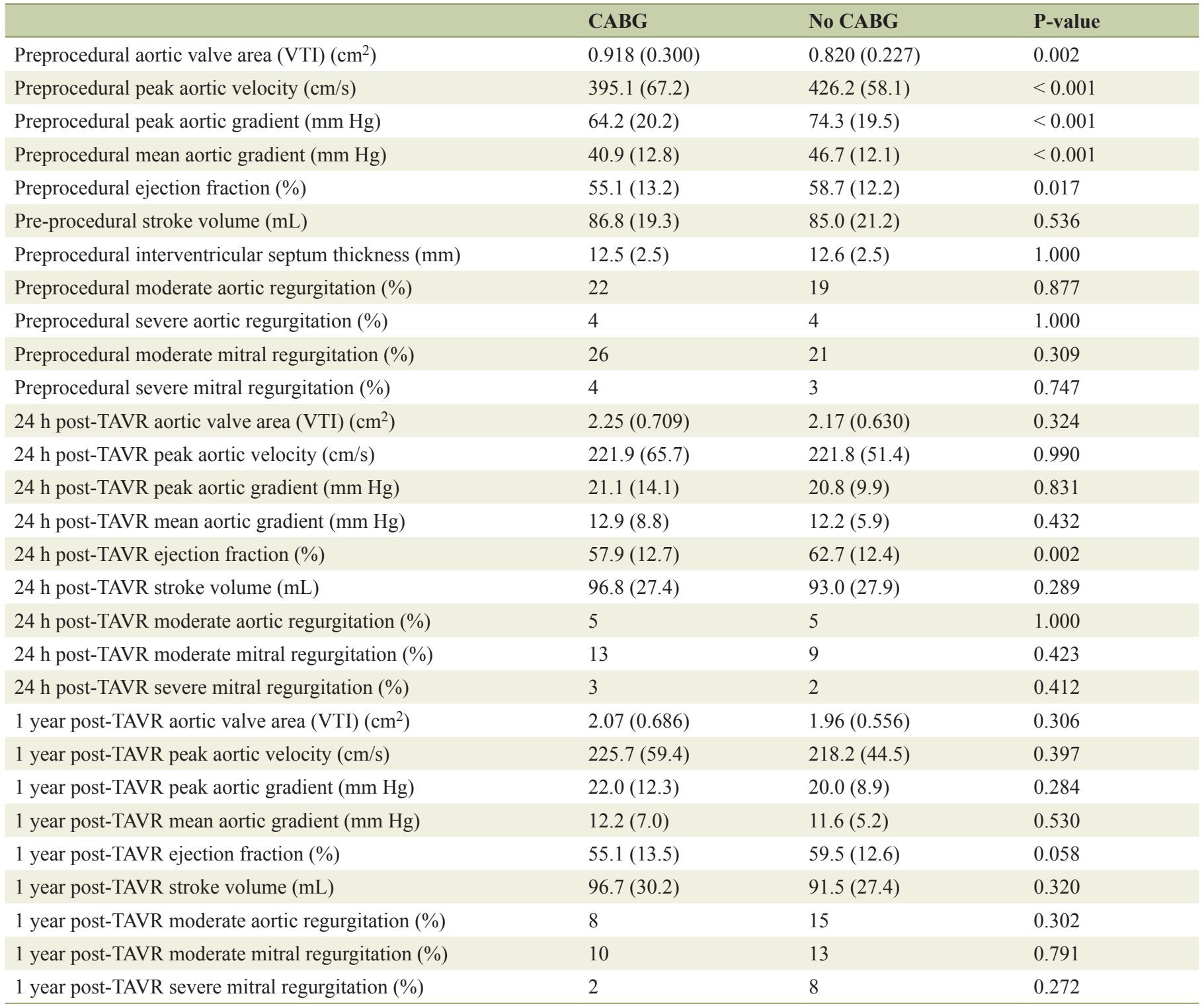

Values are mean (standard deviation) or \%.

STS risk scores suggests that traditional risk scores may not accurately reflex the true risk associated with TAVR. This has also been suggested in other previous published studies. For example, the work by Yamaoka and colleagues suggested that STS scores may overestimate the true mortality risk of TAVR [11]. On the other hand, a meta-analysis from 2014 suggested that both of these scoring algorithms underestimate the risk of TAVR [12]. Taken together, these studies and the findings of this study suggest that there needs to be a more personalized and academically more rigorous way of estimating the risk of TAVR in the periprocedural period. More studies on patients' specific factors like a history of prior $\mathrm{CABG}$ will advance the understanding of TAVR and help define which factors are true determinants of preoperative risk.

Finally, our study does have some limitations including its retrospective design, single center experiences, and variability in the length of post-TAVR follow-up. Unsurprisingly, there are some statistically significant differences in baseline characteristics, however these differences are of little clinical significance and likely do not impact this study's conclusions. Previous studies have shown that male sex may be a negative prognostic marker post-TAVR, however the effect size of this appears to be small $[9,13]$. A history of CAD regardless of severity prior to TAVR has not been associated with worse outcomes after TAVR [14]. The impact of HMG Co-A reductase inhibitors on TAVR outcomes has been studied only retrospectively to date and there is an overall paucity of data, including no data from large databases [15]. Additionally, we are not aware any data that would suggest a difference in outcomes in patients with a history of AAA or the absence of beta blocker 
Table 4. Primary and Secondary Outcomes

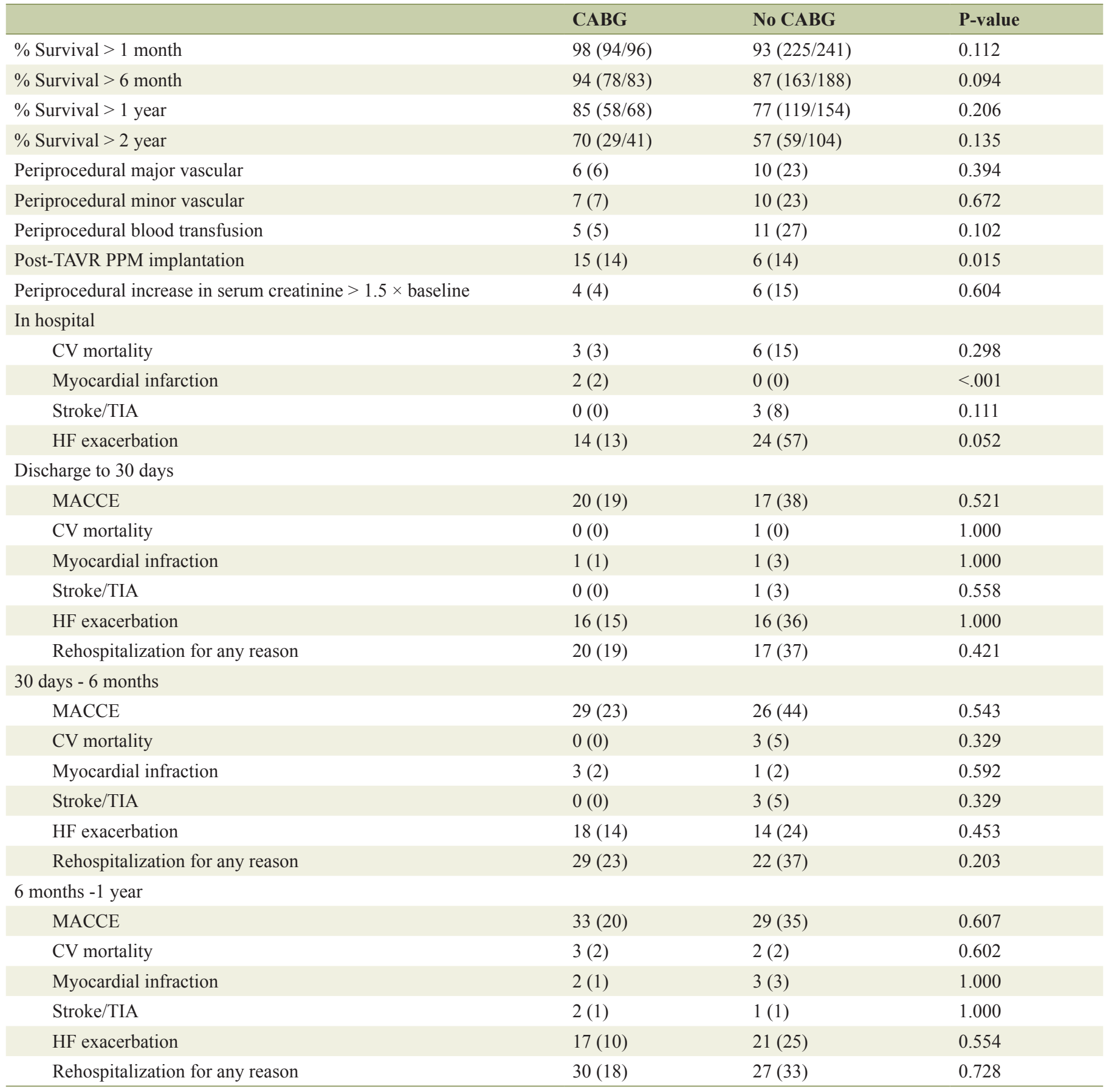

Values are \% (n). MACCE: major adverse cardiovascular and cerebrovascular events, defined as death from any cause, myocardial infarction, rehospitalization, and stroke.

or aspirin use prior to TAVR.

\section{Conclusions}

In this single center study of patients undergoing TAVR, no difference in overall survival was seen in patients with a prior history of $\mathrm{CABG}$ versus a cohort of patient without a history of CABG. A history of prior CABG was associated with an increased risk of post-TAVR PPM implantation. This study affirms the efficacy and safety of TAVR in prior CABG patients in comparison to other TAVR patients at lower predicted surgical risk. Our study also confirms the safety of TAVR in this specific population in lower volume centers. 


\section{Acknowledgments}

The investigators would like to thank Abe Sahmoun, $\mathrm{PhD}$ for his help with the statistical analysis for this study, Thomas Haldis, DO and Cornelius Dyke, MD for their help in getting this project started, and Ronda Bolgrean, $\mathrm{RN}$ for her help with data acquisition.

\section{Financial Support}

This research did not receive any specific grant from funding agencies in the public, commercial, or not-for-profit sectors.

\section{Disclosures}

This research was performed at Sanford Health Fargo, 5225 23rd Ave South, Fargo, ND 58104. The author has no relationships with industry to disclose.

\section{References}

1. Yau TM, Borger MA, Weisel RD, Ivanov J. The changing pattern of reoperative coronary surgery: trends in 1230 consecutive reoperations. J Thorac Cardiovasc Surg. 2000;120(1):156-163.

2. Reardon MJ, Van Mieghem NM, Popma JJ, Kleiman NS, Sondergaard L, Mumtaz M, Adams DH, et al. Surgical or Transcatheter Aortic-Valve Replacement in IntermediateRisk Patients. N Engl J Med. 2017;376(14):1321-1331.

3. Smith CR, Leon MB, Mack MJ, Miller DC, Moses JW, Svensson LG, Tuzcu EM, et al. Transcatheter versus surgical aortic-valve replacement in high-risk patients. N Engl J Med. 2011;364(23):2187-2198.

4. Greason KL, Mathew V, Suri RM, Holmes DR, Rihal CS, McAndrew T, Xu K, et al. Transcatheter versus surgical aortic valve replacement in patients with prior coronary artery bypass graft operation: a PARTNER trial subgroup analysis. Ann Thorac Surg. 2014;98(1):1-7; discussion 7-8.

5. Nguyen TC, Babaliaros VC, Razavi SA, Kilgo PD, Devireddy CM, Leshnower BG, Mavromatis K, et al. Transcatheter aortic valve replacement has improved short-term but similar midterm outcomes in isolated aortic valve replacement after prior coronary artery bypass grafting. Ann Thorac Surg. 2014;98(4):1316-1324.

6. Onorati F, D’Onofrio A, Biancari F, Salizzoni S, De Feo
M, Agrifoglio M, Mariscalco G, et al. Results of surgical aortic valve replacement and transapical transcatheter aortic valve replacement in patients with previous coronary artery bypass grafting. Interact Cardiovasc Thorac Surg. 2016;22(6):806-812.

7. Breen P, Lee JW, Pomposelli F, Park KW. Timing of highrisk vascular surgery following coronary artery bypass surgery: a 10-year experience from an academic medical centre. Anaesthesia. 2004;59(5):422-427.

8. Kappetein AP, Head SJ, Genereux P, Piazza N, van Mieghem NM, Blackstone EH, Brott TG, et al. Updated standardized endpoint definitions for transcatheter aortic valve implantation: the Valve Academic Research Consortium-2 consensus document. J Thorac Cardiovasc Surg. 2013;145(1):6-23.

9. Chandrasekhar J, Dangas G, Yu J, Vemulapalli S, Suchindran S, Vora AN, Baber U, et al. Sex-based differences in outcomes with transcatheter aortic valve therapy: TVT registry from 2011 to 2014. J Am Coll Cardiol. 2016;68(25):2733-2744.

10. Mauri V, Reimann A, Stern D, Scherner M, Kuhn E, Rudolph V, Rosenkranz S, et al. Predictors of permanent pacemaker implantation after transcatheter aortic valve replacement with the SAPIEN 3. JACC Cardiovasc Interv. 2016;9(21):2200-2209.

11. Yamaoka H, Kuwaki K, Inaba H, Yamamoto $T$, Kato TS, Dohi S, Matsushita S, et al. Comparison of modern risk scores in predicting operative mortality for patients undergoing aortic valve replacement for aortic stenosis. J Cardiol. 2016;68(2):135-140.

12. Biancari F, Juvonen T, Onorati F, Faggian G, Heikkinen J, Airaksinen J, Mariscalco G. Meta-analysis on the performance of the EuroSCORE II and the Society of Thoracic Surgeons Scores in patients undergoing aortic valve replacement. J Cardiothorac Vasc Anesth. 2014;28(6):15331539.

13. Arnold SV, Reynolds MR, Lei Y, Magnuson EA, Kirtane AJ, Kodali SK, Zajarias A, et al. Predictors of poor outcomes after transcatheter aortic valve replacement: results from the PARTNER (Placement of Aortic Transcatheter Valve) trial. Circulation. 2014;129(25):2682-2690.

14. Paradis JM, White JM, Genereux P, Urena M, Doshi D, Nazif T, Hahn R, et al. Impact of coronary artery disease severity assessed with the SYNTAX score on outcomes following transcatheter aortic valve replacement. J Am Heart Assoc. 2017;6(2):e005070.

15. Huded CP, Benck LR, Stone NJ, Sweis RN, Ricciardi MJ, Malaisrie SC, Davidson CJ, et al. Relation of intensity of statin therapy and outcomes after transcatheter aortic valve replacement. Am J Cardiol. 2017;119(11):1832-1838. 